\title{
Komplementärmedizin und Naturheilkunde von Anfang an - das Interesse schon während des Studiums wecken
}

\author{
Holger Cramer Heidemarie Haller Petra Klose Jost Langhorst Gustav Dobos \\ Alfried Krupp von Bohlen und Halbach-Stiftungsprofessur für Naturheilkunde, Universität Duisburg-Essen, Deutschland
}

Seit 2003 sind Leistungsnachweise im Querschnittsbereich Rehabilitation, Physikalische Medizin und Naturheilverfahren in der Bundesrepublik Deutschland verpflichtender Bestandteil der ärztlichen Ausbildung [1]. Einer aktuellen Studie zufolge wurden naturheilkundliche Inhalte bis 2010 jedoch lediglich bei $18 \%$ der deutschen medizinischen Hochschulen in die Lehre integriert [2]. Vor dem Hintergrund der stetig wachsenden Evidenzen auf dem Gebiet der Naturheilkunde und deren präventiven sowie supportiven Möglichkeiten in einer integrativen Medizin ist die bisherige Entwicklung als ungenügend einzuschätzen.

An der Universität Duisburg-Essen ist es heute schon gelungen, neben dem Besuch von Vorlesungen, die Teilnahme an einem eintägigen Praxiskurs in Naturheilkunde verpflichtend in den Lehrplan aufzunehmen. In diesem siebenstündigen Kurs erlernen und vertiefen die Studenten theoretische Grundlagen wie auch praktische Anwendungen der Europäischen Naturheilkunde, der Traditionellen Chinesischen Medizin und der Mind/Body-Medizin, wobei der Schwerpunkt der Veranstaltung auf der Vermittlung evidenzbasierten Wissens liegt. Der Seminarcharakter eröffnet den Studenten die Chance, sowohl mit naturheilkundlich praktizierenden Ärzten und Mind/Body-Therapeuten als auch mit Patienten in Dialog zu treten und Vor- und Nachteile der Therapien sowie deren Grenzen in der Praxis zu erfahren. Neben der eigenen Tätigkeit als «Behandler» können die Teilnehmer in der Rolle des «Patienten» zudem die Wirkung ausgewählter Therapieverfahren am eigenen Körper erleben. Die Kurse werden organisiert und durchgeführt von der Klinik für Naturheilkunde und Integrative Medizin, Kliniken-Essen-Mitte und der Alfried Krupp von Bohlen und Halbach-Stiftungsprofessur für Naturheilkunde an der Universität Duisburg-Essen. Sie können an mehreren Terminen im Semester besucht werden.

In einer universitätsweiten studentischen Evaluation wurde das Seminar 2010 unter 42 Pflichtkursen im Bereich klinische Medizin am besten bewertet und erhielt den Lehrpreis der Medizinischen Fakultät der Universität DuisburgEssen. Zusätzlich wurde der Kurs für das Sommersemester 2010 in einer eigenen Evaluation der Stiftungsprofessur mit dem Fragebogen für medizinische Fortbildungsveranstaltungen der Landesärztekammer Nordrhein untersucht. Die Studenten bewerteten den Kurs auf einer 6-stufigen LikertSkala nach dem Vorbild des deutschen Schulnotensystems (von 1 = sehr gut bis $6=$ ungenügend) bezüglich 7 verschiedener Kategorien (Tab. 1). Sie wurden darüber hinaus zu ihrem Interesse an Komplementärmedizin und Naturheilkunde befragt und dahingehend, ob sich dieses Interesse durch die Kursteilnahme verändert habe (die Antwortmöglichkeit war dichotom: ja/nein). Abschließend bestand Raum für Freitextkommentare, Kritik und Anmerkungen.

136 Studenten (davon 86 weiblich) von insgesamt 158 Kursteilnehmern (Rücklaufquote: 86,08\%) evaluierten den Kurs. Die Kategorien a) bis d) wurden alle mit «gut», die Kategorie e) mit «sehr gut» bewertet (Abb. 1). Weibliche Studenten vergaben bessere Noten bezüglich erfüllter Erwartungen $(p=0,039)$ und hinsichtlich der Frage, ob der Kurs sich gelohnt habe $(\mathrm{p}=0,029)$, während sie schlechtere Noten bezüglich ähnlichen Lernerfolgs durch die neuen Medien $(\mathrm{p}=0,034)$ vergaben (Abb.1). Studenten, die Interesse an Komplementärmedizin und Naturheilkunde äußerten (76,5\%), gaben bessere Noten bezüglich erfüllter Erwartungen $(p<0,001)$, Lernerfolg $(p=0,027)$ und Praxisrelevanz ( $\mathrm{p}<0,001$ ) (Abb.1). 44,1\% der Studenten gaben an, dass sich ihr Interesse an Komplementärmedizin und Naturheilkunde durch die Kursteilnahme verändert hat.

Einige Freitextkommentare weisen ebenfalls auf einen Wandel im Interesse der Studenten hin. So wurde das Seminar als «überraschend positive Veranstaltung, durch die ich mich motiviert fühle, für Naturheilkunde offen zu bleiben» beschrieben. Die Studenten schilderten den Kurs als «wirklich

\section{KARGER}

Fax +497614520714

Information@Karger.de

www.karger.com (c) 2011 S. Karger GmbH, Freiburg

1661-4119/11/0186-0357\$38.00/0

Accessible online at:

www.karger.com/fok
Holger Cramer

Alfried Krupp von Bohlen und Halbach-Stiftungsprofessur für Naturheilkunde,

Universität Duisburg-Essen, Klinik für Naturheilkunde und Integrative Medizin

Kliniken Essen-Mitte, Knappschafts-Krankenhaus

Am Deimelsberg 34a, 45276 Essen, Deutschland, Tel. +49 201 17425-054, Fax -000

h.cramer@kliniken-essen-mitte.de 
Tab. 1. Kategorien des Fragebogens und die jeweilige exakte Fragestellung

\begin{tabular}{ll}
\hline Kategorie & Frage \\
\hline a) Erwartungen erfüllt & Meine Erwartungen hinsichtlich der Ziele und Themen des Kurses haben sich erfüllt \\
b) Gelernt & Während des Kurses habe ich gelernt \\
c) Praktische Relevanz & Der Kurs hat meiner Meinung nach Relevanz für meine praktische ärztliche Tätigkeit \\
d) Lohnenswert & Gemessen am zeitlichen und organisatorischen Aufwand hat sich die Teilnahme an diesem \\
& Kurs für mich gelohnt \\
e) Diskussion & Während des Kurses bestand ausreichend Gelegenheit zur Diskussion, meine Fragen \\
f) Lektüre & wurden beantwortet \\
g) Neue Medien & Mit gleichem Zeitaufwand hätte ich einen vergleichbaren Gewinn erreicht durch Lektüre \\
\hline
\end{tabular}

eindrückliches Erlebnis», «horizonterweiternd» und «die eigene Lebensweise überdenkend» und lobten, dass der Kurs «sehr anschaulich und praxisorientiert» war, sie «besonders die praktischen Übungen überzeugt» hätten, und sie fanden es «gut, diese Anwendungen einmal selbst zu erleben». Für verbesserungswürdig hielten die Studenten «Handouts mit den basic essentials» und gaben an, die «Wirkmechanismen müssten besser erklärt werden».

Zusammenfassend wurde ein praxisnahes Pflichtseminar mit dem Schwerpunkt auf evidenzbasierter Komplementärmedizin von Medizinstudenten als «gut» bis «sehr gut» sowie lohnend und bedeutsam für die spätere ärztliche Tätigkeit bewertet. Die Rücklaufquote war dabei mit der anderer Lehrevaluationen im Fachbereich Medizin vergleichbar [3]. Weibliche Teilnehmer evaluierten den Kurs als eher den Erwartungen entsprechend, lohnenswerter und weniger durch neue Medien substituierbar. Studenten, die nach dem Kurs ein Interesse an Komplementärmedizin bekundeten, schätzten den Lernerfolg und die Praxisrelevanz höher ein. Fast die Hälfte der Studenten gab an, ihr Interesse habe sich durch die Kursteilnahme verändert, so dass nach dem Kurs mehr als drei Viertel der Studenten Interesse an Komplementärmedizin bekundeten. Im Gegensatz zu bisherigen Studien [4] deutet dies darauf hin, dass eine praxisnahe Vermittlung evidenzbasierter Komplementärmedizin bei Medizinstudenten, die sich zunächst nicht für Komplementärmedizin interessieren, das Interesse für diese Therapieformen steigern kann. Insbe-

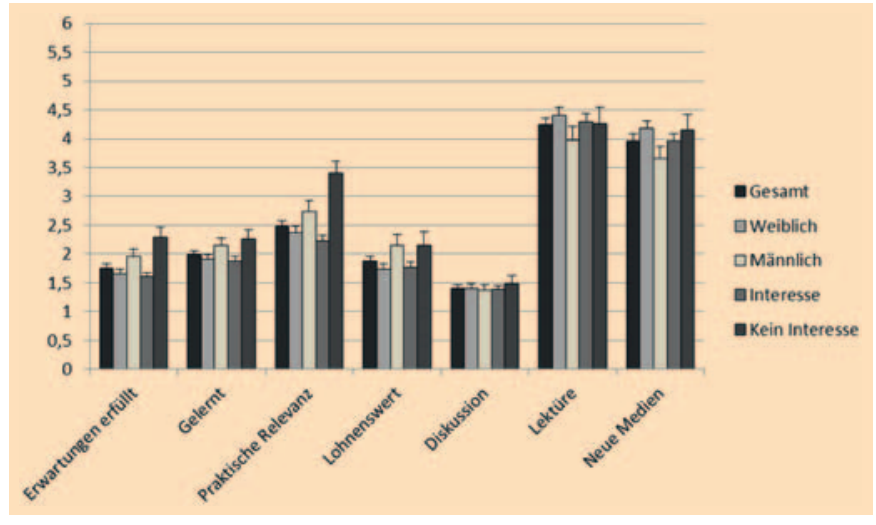

Abb. 1. Ergebnisse der Lehrevaluation. Dargestellt ist der Mittelwert mit dem Standardfehler für die gesamte Stichprobe sowie getrennt nach Geschlecht und Interesse an Naturheilkunde und Komplementärmedizin. Niedrigere Noten drücken höhere Zustimmung aus. Bei der Frage nach Lektüre und neuen Medien bedeuten höhere Noten demnach eine geringere Substituierbarkeit der Kursinhalte durch diese Medien.

sondere die praktische Erprobung des Gelernten scheint einen wichtigen Bestandteil dieses Prozesses darzustellen und kann als ein gutes Modell für die Integration von Komplementärmedizin und Naturheilkunde bereits während des Studiums dienen. Denn für die Zukunft der Komplementärmedizin wird es am wichtigsten sein, wie heutige Medizinstudenten ihr gegenüberstehen [5].

\section{Literatur}

1 Jobst D, Musselmann B: Naturheilverfahren - ein Publikumsliebling geht an die Uni. Z Allgemeinmed 2003;79:605-608.

2 Brinkhaus B, Witt CM, Jena S, Bockelbrink A, Ortiz $\mathrm{M}$, Willich SN: Integration of complementary and alternative medicine into medical schools in Austria, Germany and Switzerland - results of a cross-sectional study. Wien Med Wochenschr 2010;161:32-43.
3 Pabst R, Nave H, Rothkötter HJ, Tschernig T: Lehrevaluation in der Medizin. Befragung zur Qualität der Lehre in den Hochschulen. Dtsch Ärztebl 2001;98:747-749.

4 Nicolao M, Täuber MG, Marian F, Heusser P: Complementary medicine courses in Swiss medical schools: actual status and students' experience. Swiss Med Wkly 2010;140:44-51.
5 Freedman DH: The triumph of new-age medicine. Atlantic, 2011. www.theatlantic.com/magazine/archive/ 2011/07/the-triumph-of-new-age-medicine/8554/. 